\title{
Importancia de las clases de laboratorio en la motivación de los alumnos de la asignatura Materiales Construcción Importance of laboratory classes in motivating students in the Construction Materials course
}

\author{
Departamento de Ingeniería Civil: Construcción \\ Universidad Politécnica de Madrid \\ Madrid, España
}

Ana Patricia Pérez-Fortes, Jaime C. Gálvez, Encarnación Reyes, Alejandro Enfedaque

anapatricia.perez@upm.es, jaime.galvez@upm.es, encarnacionreyes@upm.es, alejandro.enfedaque@upm.es

\begin{abstract}
Resumen- Durante el curso 2020/2021 se han retomado las clases de laboratorio de la asignatura de Materiales de Construcción I del grado de Ingeniería Civil y Territorial de la ETSI de Caminos, Canales y Puertos de la Universidad Politécnica de Madrid. Estas clases, de carácter voluntario, se han impartido en los tres grupos de la asignatura. Los resultados de la experiencia han sido contrastados con los datos del curso 2018/2019, debido a la excepcionalidad del curso 2019/2020, afectado por la pandemia del COVID 19. Además, se ha realizado una encuesta voluntaria a los participantes de las clases de laboratorio para conocer su motivación para asistir a estas clases y sus impresiones sobre ellas. El $70 \%$ de los estudiantes matriculados en la asignatura durante el curso 2020/2021 han asistido voluntariamente a las clases de laboratorio y realizado los trabajos exigidos con la intención de mejorar la nota de la asignatura. Sin embargo, durante el desarrollo de las clases, su interés aumentó, lo que se ha traducido en el aumento de la participación de los estudiantes en la modalidad de evaluación continua de la asignatura, disminuyendo, igualmente, el abandono de esta modalidad durante este curso con relación al curso 2018/2019.
\end{abstract}

Palabras clave: Clases de laboratorio, evaluación continua, ingeniería civil y territorial.

\begin{abstract}
During the 2020/2021 academic year, the laboratory classes of the Construction Materials I subject of the Civil and Territorial Engineering degree of the Civil Engineering School at the Technical University of Madrid have been restarted. These classes, of a voluntary nature, have been taught in the three groups of the subject. The results of the experience have been contrasted with the data for the 2018/2019 academic year, due to the exceptional nature of the 2019/2020 academic year, affected by the COVID 19 pandemic situation. In addition, a voluntary survey has been carried out among the participants of the laboratory classes to find out their motivation for attending these classes and their impressions of them. $70 \%$ of the students enrolled in the course during the 2020/2021 academic year have voluntarily attended the laboratory classes and carried out the required work with the intention of being able to raise their scores. However, during the development of the classes, their interest increased, which has resulted in an increase in the participation of the students in the continuous assessment modality of the subject, also decreasing the abandonment of this modality during this course in relation to the 2018/2019 academic year.
\end{abstract}

Keywords: Laboratory classes, continuous evaluation, civil and territorial engineering.

\section{INTRODUCCIÓN}

Las clases de laboratorio son una parte esencial del aprendizaje en ingeniería, reforzando la teoría a través de la experiencia práctica (Surgenor and Firth, 2006; Boxal and Tait, 2008; Temmen et al., 2014; Restivo et al., 2018; Khabiri and Bahabad, 2019; Molvinger et al., 2020). Sin embargo, el elevado número de estudiantes, el aumento de la carga de trabajo de los estudiantes y la necesidad de disponer de los equipos adecuados y de personal técnico cualificado dificultan ofertar este tipo de clases durante el curso académico (Boxal and Tait, 2008; McCabe et al., 2019). Por estos motivos, las universidades tienden cada vez más a la digitalización de este tipo de enseñanza experimental o a su sustitución por otras actividades.

Generalmente, en los primeros años de ingeniería, los estudiantes sólo aspiran a aprobar las asignaturas que cursan, frente al deseo de acumular los conocimientos necesarios para el desarrollo de la profesión o de profundizar en las materias ofertadas (Reyes et al., 2021), lo que dificulta aún más la implicación del estudiante en actividades extracurriculares.

Sin embargo, las clases de laboratorio sirven para reforzar los conceptos que se tratan en las clases teóricas, permite a los estudiantes comparar los resultados experimentales con la teoría y relacionar los experimentos y los resultados con aplicaciones en el mundo real y les obliga a lidiar con la incertidumbre (error experimental, McCabe et al., 2019). De este modo, además de ayudar a la comprensión de la asignatura, el estudiante se sentirá atraído por todo aquello que tenga una aplicación real y directa (Reyes et al., 2021).

Por estos motivos, se ha considerado necesario recuperar las clases de laboratorio en la asignatura de Materiales de Construcción I, impartida en el primer semestre del segundo año de grado de Ingeniería Civil y Territorial de la ETSI de Caminos, Canales y Puertos. Para ello, durante la preparación y desarrollo de las clases, se ha hecho hincapié, no sólo en reforzar las clases teóricas de la asignatura, sino también otras competencias transversales tales como la reflexión y síntesis de los datos experimentales o las habilidades informáticas. Además, durante el desarrollo de las clases, se ha incidido 
especialmente en las aplicaciones de los experimentos y ensayos propuestos en obra civil.

\section{CONTEXTO}

Durante la reestructuración en 2016 de la asignatura de Materiales de Construcción I, impartida en el segundo año del grado de Ingeniería Civil y Territorial de la ETSI de Caminos, Canales y Puertos, se procedió a eliminar las clases de laboratorio, instaurándose un nuevo sistema de evaluación continua. Dicho sistema consiste en la realización, tanto en clase como en casa, de problemas y simulacros de examen que son calificados por el profesor durante el semestre que dura la asignatura. De este modo, se realiza un seguimiento del estudiante a lo largo del curso que también le ayuda a prepararse para los exámenes parciales y finales de la asignatura.

En el curso 2020/2021, sin embargo, se ha querido reforzar la metodología de evaluación continua de la asignatura, volviéndose a instaurar las clases de laboratorio. Estas clases son de carácter voluntario y duran 1 h $\quad$ y 30 min aproximadamente. Para motivar a los estudiantes a participar en las mismas, la nota de estas prácticas suma hasta un punto extra en la nota de la evaluación continua, en función de la calificación obtenida en los trabajos de laboratorio exigidos, sin tener ninguna penalización por el suspenso en los mismos.

En este trabajo, por tanto, se analizará la influencia de la recuperación de las clases de laboratorio de materiales de construcción en la participación de los estudiantes en la modalidad de evaluación continua, así como en las calificaciones finales de la asignatura. Para ello, se analizarán las notas obtenidas por los estudiantes matriculados en los cursos 2018/2019 y 2020/2021, siendo el año académico 2018/2019 el "curso de control". Se ha descartado el empleo del año académico 2019/2020 como "curso de control" debido a la situación excepcional ocurrida por la pandemia del COVID19.

Además, para completar la información sobre el impacto de las clases de laboratorio en la participación de los estudiantes en la asignatura, se ha realizado una breve encuesta de carácter voluntario a través de la plataforma Moodle. En esta encuesta, los estudiantes de forma anónima han respondido a una serie de preguntas sobre sus impresiones acerca de las clases impartidas, su motivación para asistir y las competencias adquiridas mediante la entrega de trabajos.

\section{DESCRIPCIÓN}

Como se ha mencionado en el apartado anterior, cada clase de laboratorio dura aproximadamente $1 \mathrm{~h}$ y $30 \mathrm{~min}$ y se realiza en grupos pequeños, de 14 personas aproximadamente. En total, cada estudiante asiste a dos clases de laboratorio durante el semestre, teniendo que entregar un trabajo sobre la actividad desarrollada en el laboratorio después de cada clase.

Por tanto, la metodología de trabajo en el laboratorio puede dividirse en 4 etapas: organización de los grupos de clase y del material necesario para el desempeño del trabajo de laboratorio; trabajo experimental de laboratorio y recopilación de datos; realización de un trabajo o informe de laboratorio; evaluación y autorreflexión sobre los trabajos entregados (Figura 1).
Organización de los grupos

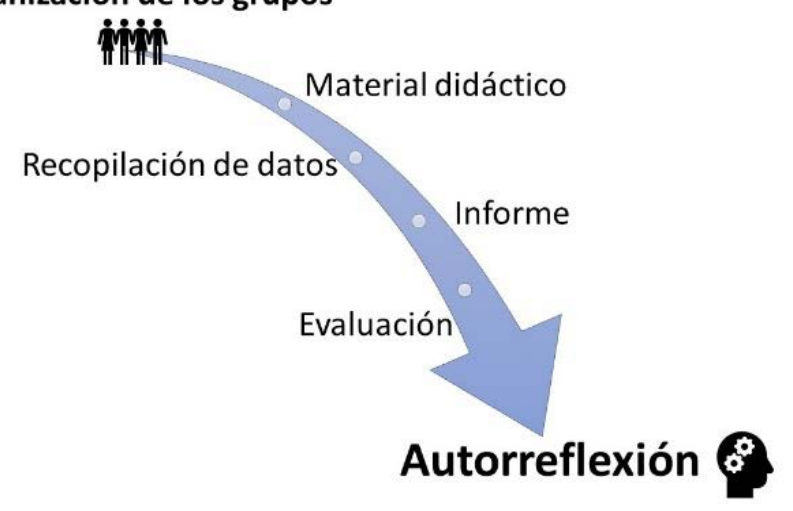

Figura 1: Esquema de la metodología aplicada para el desarrollo de las clases de laboratorio.

\section{A. Organización de los grupos de clase y del material necesario para el desempeño del trabajo de laboratorio}

Antes de comenzar las clases de laboratorio, se dio a conocer durante las clases teóricas de la asignatura su existencia y la posibilidad de asistir de forma voluntaria a las mismas. Igualmente, se explicó el sistema de evaluación de cada práctica de laboratorio y su peso en la nota de clase (evaluación continua) y la nota final.

Debido a la elevada participación de los estudiantes (174 estudiantes asistieron a prácticas de los 247 matriculados) y al poco espacio disponible en el laboratorio, se tuvieron que organizar 13 grupos de aproximadamente 14 personas. Para ello, se generaron en Moodle grupos donde los estudiantes pudieron elegir día y hora para asistir a las clases.

Igualmente, se pusieron a disposición de los estudiantes en Moodle guiones de prácticas con la teoría necesaria para poder realizar los trabajos de laboratorio exigidos y fichas de laboratorio para facilitar la toma de datos.

\section{B. Trabajo experimental de laboratorio y recopilación de datos}

Una vez organizados los grupos de prácticas y con el material disponible en Moodle, los estudiantes asistieron al laboratorio, donde se realizaron diferentes experimentos y ensayos con diferentes materiales de construcción. Los ensayos y materiales de construcción utilizados fueron seleccionados de acuerdo con la teoría explicada en clase, con la finalidad de reforzar los conocimientos teóricos adquiridos.

Durante el desarrollo de cada clase de laboratorio, los estudiantes, de forma voluntaria, o realizaban el ensayo o asistían al técnico encargado durante el mismo, dependiendo de la dificultad de manejo de los equipos utilizados y las medidas de seguridad pertinentes. Dichos voluntarios, además, eran los encargados de facilitar a sus compañeros los datos necesarios para poder realizar el trabajo de laboratorio en casa. Con esta metodología, los estudiantes se familiarizaron de forma directa con el trabajo que se desempeña en un laboratorio de materiales de construcción. Además, ayudó a que se responsabilizaran, desde el inicio de la clase, de los trabajos que debían entregar, desde la toma de datos hasta su procesado y obtención de resultados y conclusiones. 
Los datos recopilados por los estudiantes en estas clases fueron, desde sencillas medidas tomadas con una balanza hasta hojas de cálculo con cientos de datos procedentes de las máquinas de ensayos de resistencia a tracción disponibles en el laboratorio. De este modo, el grado de complejidad en el tratamiento y análisis de los datos aumentó a medida que avanzaba el curso.

\section{Realización de un trabajo o informe de laboratorio}

Una vez hecha la toma de datos en el laboratorio, los estudiantes debieron realizar una tarea a través de la plataforma Moodle. En esta tarea, además de los cálculos necesarios para poder obtener determinadas propiedades de los materiales de construcción, debían responder preguntas cortas sobre dichas propiedades, con el fin de afianzar los conceptos teóricos aprendidos en clase de una forma experimental.

Además de tener como objetivo reforzar los conceptos teóricos de la asignatura, con la entrega de estas tareas se ha intentado trabajar en otras competencias transversales de los estudiantes, tales como la reflexión y síntesis de los datos recopilados en el laboratorio, la organización del tiempo (imposición de fecha de entrega de los trabajos) o las habilidades informáticas (manejo de herramientas de procesamiento de texto y hojas de cálculo).

\section{Evaluación y autorreflexión sobre los trabajos entregados}

Como se ha mencionado en el apartado anterior, los trabajos de laboratorio fueron entregados en forma de tarea a través de la plataforma Moodle. De esta forma, los estudiantes pudieron recibir en forma de comentarios privados y personalizados la información sobre los errores cometidos.

La privacidad y personalización de los comentarios tiene como finalidad que cada estudiante pueda hacer una autorreflexión sobre los puntos teórico-prácticos que debe reforzar en la asignatura.

\section{Resultados}

Durante el curso 2018/2019, el 63 \% de los estudiantes matriculados en la asignatura de Materiales de Construcción I se presentaron al examen final, de los cuales, aprobó el $65 \%$ (Figura 2). Por el contrario, la participación en este examen aumentó en el curso 2020/2021 hasta un $81 \%$, aunque sólo el $63 \%$ de los estudiantes presentados aprobaron la asignatura (Figura 2). Este aumento en la participación en el examen final está también acompañado de un aumento en el seguimiento del sistema de evaluación continua por parte de los estudiantes matriculados durante el curso 2020/2021 (un $72 \%$ frente al 60 $\%$ de participación en el curso 2018/2019, véase Figura 3). Cabe mencionar que el abandono del sistema de evaluación continua por parte de los estudiantes durante el curso 2020/2021 disminuyó casi a la mitad, del $32 \%$ al $18 \%$.

Estos datos sugieren, por tanto, que, aunque la posibilidad de realizar prácticas de laboratorio no ha tenido un impacto significativo en el porcentaje de aprobados finales de la asignatura, sí que ha influido positivamente en la participación de los estudiantes en el sistema de evaluación continua de la asignatura, disminuyendo, el nivel de abandono en la misma.
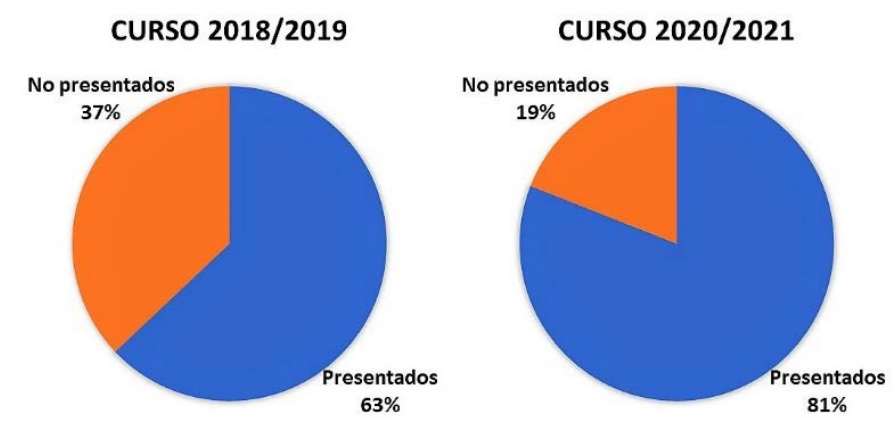

Figura 2: Relación de estudiantes presentados y no presentados al examen final de la asignatura de Materiales de Construcción I durante los cursos 2018/2019 y 2020/2021.
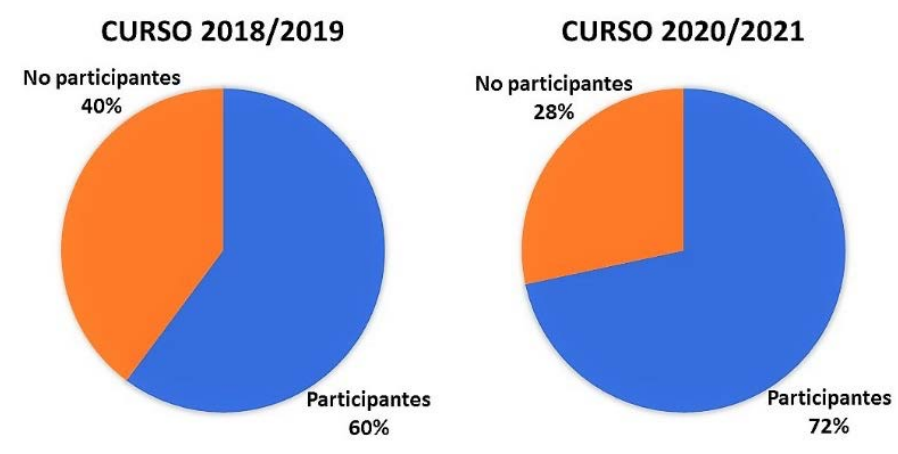

Figura 3: Relación de estudiantes que participaron en el sistema de evaluación continua de la asignatura en los cursos 2018/2019 y 2020/2021.

Además, se procedió a realizar una breve encuesta de satisfacción para conocer mejor la motivación de los estudiantes a participar en las clases de laboratorio y su grado de satisfacción una vez realizadas. En esta encuesta, participaron de forma voluntaria 73 estudiantes, de los 180 que asistieron a las clases prácticas. A pesar de ser una actividad voluntaria, el $70 \%$ de los estudiantes matriculados en la asignatura participaron en las prácticas. Esto, según los estudiantes encuestados fue debido a que la nota obtenida en las mismas podría ayudarles a aprobar (56\%). En menor medida, el $41 \%$ sentía curiosidad por conocer el trabajo de laboratorio relacionado con los materiales de construcción, mientras que un $3 \%$ tenía otros motivos. Sin embargo, una vez realizadas las clases de laboratorio, el $71 \%$ de los estudiantes encuestados afirmaron tener un interés alto por este tipo de clases, un $26 \%$ un interés medio y sólo un $3 \%$ un interés bajo. Igualmente, el $93 \%$ de los estudiantes encuestados piensan que su participación en las clases de laboratorio les ha ayudado a entender mejor la asignatura, un $48 \%$ cree que estas clases les ha ayudado a desarrollar otras capacidades tales como la reflexión y síntesis de datos, un $45 \%$ opina que han mejorado sus habilidades informáticas y sólo un $18 \%$ cree que ha mejorado su capacidad de organización del tiempo. Estos resultados indican que, aunque la motivación inicial de los estudiantes fuera la de subir su nota, durante el desarrollo de las clases de laboratorio y, con la metodología propuesta, aumentó su interés por este tipo de clases. De igual modo, los estudiantes han sido conscientes del desarrollo de otras competencias transversales al asistir al laboratorio y entregar los trabajos correspondientes, como son el tratamiento y análisis de datos experimentales o el uso de herramientas informáticas. Todo ello, junto con la importancia del seguimiento de las clases, sólo 
el $9 \%$ y el $4 \%$ de los alumnos presentados al examen final aprobó la asignatura sin seguir la evaluación continua en los cursos 2018/2019 y 2020/2021 respectivamente (Figura 4), ha podido servir para captar la atención del estudiante, incrementando su participación en clase y en los exámenes finales.

\section{CURSO 2018/2019}
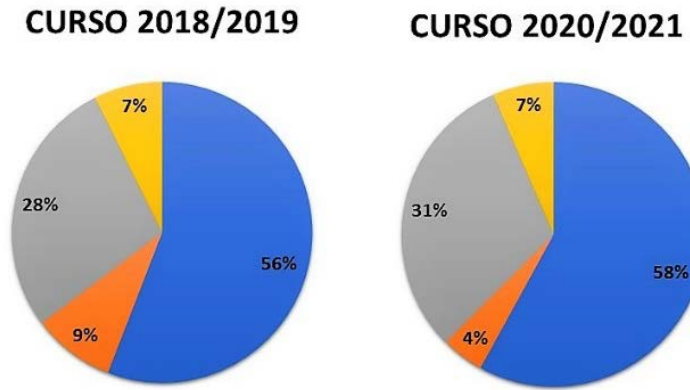

- Aprobado con evaluación continua $₫$ Aprobado sin evaluacion continua u Suspenso con evaluacion continua $\quad$ Suspenso sin evaluacion continua

Figura 4: Proporción de estudiantes presentados al examen final de la asignatura que aprobaron o suspendieron, en función de su seguimiento del sistema de evaluación continua.

\section{CONCLUSIONES}

Durante el curso académico 2020/2021, se ha ofrecido a los estudiantes de segundo grado de Ingeniería Civil y Territorial de la ETSI de Caminos, Canales y Puertos que cursaban la asignatura de Materiales de Construcción I en el primer semestre la posibilidad de asistir de forma voluntaria a clases de laboratorio. De esta experiencia se ha concluido que:

- La proporción de estudiantes aprobados y suspensos que se presentaron al examen final de la asignatura no varió con respecto a cursos anteriores. Sin embargo, en el curso 2020/2021 aumentó el porcentaje de estudiantes que se presentaron al examen y que siguieron el sistema de evaluación continua de la asignatura.

- La elevada participación de los estudiantes en las clases de laboratorio fue motivada principalmente por la posibilidad de subir la nota de la asignatura, aunque durante el desarrollo de las clases aumentó su interés por éstas.

- Aunque la mayor parte de los asistentes a las clases de laboratorio opina que estas clases fueron útiles para reforzar los conocimientos teóricos de la asignatura, entre el 45 y el $48 \%$ de los estudiantes reconoce que su asistencia le ha ayudado a mejorar otras competencias como son el análisis y síntesis de datos o las habilidades informáticas.

- El buen recibimiento de las clases de laboratorio por parte de los estudiantes, junto con el aumento de su participación en el sistema de evaluación continua y en el examen final, son indicadores de que dichas clases pueden ser un atractivo para "enganchar" a los estudiantes a la asignatura.

\section{AgRADECIMIENTOS}

Los autores quieren agradecer a los estudiantes de la asignatura de Materiales de Construcción I del grado de Ingeniería Civil y Territorial de la ETSI de Caminos, Canales y Puertos de la Universidad Politécnica de Madrid su participación, tanto en las clases de laboratorio, como en la encuesta sobre dichas clases.

\section{REFERENCIAS}

Boxall, J., \& Tait, S. (2008, August). Inquiry-based learning in civil engineering laboratory classes. In Proceedings of the Institution of Civil Engineers-Civil Engineering, 161 (3), 138-143.

Khabiri, M. M., \& Bahabad, M. J. A. (2019). Teaching and learning of practical skills: Learning from the pavement laboratory course. Journal of Technical Education and Training, 11(2), 15-22.

McCabe, B., Pantazidou, M., \& Phillips, D. (Eds.). (2019). Shaking the Foundations of Geo-Engineering Education. CRC Press.

Molvinger, K., Ayral, R. M., \& Filhol, J. S. (2020). Integrating lecture and laboratory work for a materials chemistry course to engage and motivate students through highly visual and intriguing syntheses. Journal of Chemical Education, 97(3), 866-872.

Restivo, M. T., de Fátima Chouzal, M., Abreu, P., \& Zvacek, S. (2018, September). The role of an experimental laboratory in engineering education. In International Conference on Interactive Collaborative Learning, 644652 .

Reyes, E., Gálvez, J. C., \& Enfedaque, A. (2021). Learning Course: Application of Gamification in Teaching Construction and Building Materials Subjects. Education Sciences, 11(6), 287.

Surgenor, B., \& Firth, K. (2006). The role of the laboratory in design engineering education. Proceedings of the Canadian Engineering Education Association (CEEA), 287-293.

Temmen, K., Nofen, B., \& Wehebrink, M. (2014). Lecture meets laboratory experimental experiences for large audiences: Concept and implementation. In 2014 IEEE Global Engineering Education Conference (EDUCON), 357-360. 